PROCEEDINGS OF THE

AMERICAN MATHEMATICAL SOCIETY

Volume 125, Number 8, August 1997, Pages 2481-2484

S 0002-9939(97)03821-5

\title{
THE HOMOLOGY OF THE SPACE OF AFFINE FLAGS CONTAINING A NILPOTENT ELEMENT
}

\author{
E. SOMMERS
}

(Communicated by Roe W. Goodman)

\begin{abstract}
We show that the homology of the space of Iwahori subalgebras containing a nilpotent element of a split semisimple Lie algebra over $\mathbf{C}((\varepsilon))$ is isomorphic to the homology of the entire affine flag manifold.
\end{abstract}

\section{INTRODUCTION}

Let $G$ be a semisimple, simply connected algebraic group over $\mathbf{C}$ with Lie algebra g. Let $F=\mathbf{C}((\varepsilon)), \hat{G}=G(F)$, and $\hat{\mathfrak{g}}=\mathfrak{g} \otimes_{\mathbf{C}} F$. The affine flag manifold of $\hat{G}$, denoted $\hat{\mathcal{B}}$, is the space of Iwahori subalgebras of $\hat{\mathfrak{g}}$. This is an infinite dimensional algebraic variety (i.e. a direct limit of complex varieties under closed embeddings) with a natural action of $\hat{G}$ given by conjugation.

We recall some facts about $\hat{\mathcal{B}}$. Let $\hat{\mathfrak{b}}_{0}$ be an Iwahori subalgebra and let $\hat{B}_{0}$ denote the subgroup of $\hat{G}$ which normalizes $\hat{\mathfrak{b}}_{0}$. Let $\hat{W}$ be the affine Weyl group of $G$. This is a Coxeter group with length function $l(w)$ for $w \in \hat{W}$ and standard partial order $\leq$. Viewing $\hat{W}$ as the quotient of a subgroup $\mathfrak{M}$ of $\hat{G}$ defined in [2], we denote by $\dot{w} \in \mathfrak{M}$ a representative of $w \in \hat{W}$. For each $w \in \hat{W}$, we let $\hat{\mathcal{B}}_{w}=\hat{B}_{0} \dot{w} \hat{\mathfrak{b}}_{0} \subset \hat{\mathcal{B}}$. Then $\hat{\mathcal{B}}=\bigcup \hat{\mathcal{B}}_{w}$, where the disjoint union is over all $w$ in $\hat{W}$. This is the 'Bruhat' decomposition of $\hat{\mathcal{B}}[2]$. Furthermore, $\hat{\mathcal{B}}_{w}$ is isomorphic to $\mathbf{C}^{l(w)}$ and $\overline{\hat{\mathcal{B}}}_{w}$, the closure of $\hat{\mathcal{B}}_{w}$ in $\hat{\mathcal{B}}$, is a complex projective variety which is the union of all $\hat{\mathcal{B}}_{w^{\prime}}$ such that $w^{\prime} \leq w$. Also $\hat{\mathcal{B}}=\lim _{\underline{\hat{\mathcal{B}}}}$ (see $\left.[4]\right)$

Let $N$ be a nilpotent element of $\hat{\mathfrak{g}}$. Let $\hat{\mathcal{B}}_{N}$ be the subspace of $\hat{\mathcal{B}}$ consisting of Iwahori subalgebras containing $N$. Our main result is that the singular homology with integer coefficients of $\hat{\mathcal{B}}_{N}$ is isomorphic to the homology of $\hat{\mathcal{B}}$.

\section{KEY LEMMAS}

Lemma 1. Let $N \in \hat{\mathfrak{g}}$ be nilpotent. For any positive integer $k$, the elements $N$ and $\varepsilon^{2 k} N$ are conjugate under $\hat{G}$.

Proof. Since $\hat{G}$ is split over $F$, the Jacobson-Morozov theorem [3] implies that $N$ belongs to an $s l_{2}(F)$-subalgebra of $\hat{\mathfrak{g}}$. Now the lemma just follows from the case of $\hat{G}=S L_{2}(F)$.

Received by the editors December 20, 1995 and, in revised form, February 12, 1996.

1991 Mathematics Subject Classification. Primary 58B25.

Key words and phrases. Affine flag manifolds.

Research supported by the NSF. 
Thus for any positive integer $k$, we see that $\hat{\mathcal{B}}_{N}$ and $\hat{\mathcal{B}}_{\varepsilon^{2 k} N}$ are homeomorphic.

Now we wish to put coordinates on the space $\hat{\mathcal{B}}_{w}=\hat{B}_{0} \dot{w} \hat{\mathfrak{b}}_{0}$ for $w \in \hat{W}$. First we need some more preliminaries. Choose $\mathfrak{h}$ a Cartan subalgebra of $\mathfrak{g}$. Let $\Delta$ be the set of roots defined by $\mathfrak{h}$ and let $\Delta=\Delta^{+} \cup \Delta^{-}$be a decomposition of $\Delta$ into positive and negative roots. Let $\tilde{\Delta}=\{(\alpha, n) \mid \alpha \in \Delta$ and $n \in \mathbb{Z}\}$ and let $\tilde{\Delta}^{+}=\left\{(\alpha, n) \in \tilde{\Delta} \mid \alpha \in \Delta^{+}\right.$and $n>0$ or $\alpha \in \Delta^{-}$and $\left.n \geq 0\right\}$.

For $\alpha \in \Delta$, let $e_{\alpha}$ be a non-zero element in the root space of $\mathfrak{g}$ corresponding to $\alpha$. For $t \in F$, let $x_{\alpha}(t)=\exp \left(t e_{\alpha}\right)$ which is a well-defined element of $\hat{G}$. Now we fix $\hat{\mathfrak{b}}_{0}$ to be the Iwahori subalgebra spanned as a $\mathbf{C}$-vector space by $\mathfrak{h} \otimes \mathbf{C}[[\varepsilon]]$ and the elements $\varepsilon^{n} e_{\alpha}$ for all $(\alpha, n) \in \tilde{\Delta}^{+}$. Thus for $(\alpha, n) \in \tilde{\Delta}^{+}$and $t \in \mathbf{C}$, we know that $x_{\alpha}\left(t \varepsilon^{n}\right) \in \hat{B}_{0}$.

Now let $\tilde{\Delta}(w)=\left\{(\alpha, n) \in \tilde{\Delta}^{+} \mid \dot{w}^{-1} x_{\alpha}\left(\varepsilon^{n}\right) \dot{w} \notin \hat{B}_{0}\right\}$. It is known that the cardinality of $\tilde{\Delta}(w)$ is just $l(w)[2]$. To simplify notation, let $l$ equal $l(w)$. For $1 \leq j \leq l$, let $\left(\alpha_{j}, n_{j}\right)$ be the elements of $\tilde{\Delta}(w)$ in some order. Then from results in $[2]$, it follows that the map $\phi: \mathbf{C}^{l} \rightarrow \hat{\mathcal{B}}_{w}$ given by

$$
\phi\left(t_{1}, t_{2}, \ldots, t_{l}\right) \rightarrow x_{\alpha_{1}}\left(t_{1} \varepsilon^{n_{1}}\right) x_{\alpha_{2}}\left(t_{2} \varepsilon^{n_{2}}\right) \ldots x_{\alpha_{l}}\left(t_{l} \varepsilon^{n_{l}}\right) \dot{w} \hat{\mathfrak{b}}_{0}
$$

is an isomorphism. We use these coordinates in the following lemma.

Lemma 2. Let $N \in \hat{\mathfrak{b}}_{0}$ be any element and let $k$ be a positive integer. If $\hat{\mathcal{B}}_{\varepsilon^{k} N} \cap$ $\hat{\mathcal{B}}_{w} \neq \emptyset$, then for some complex variety $V$ and some $d \geq \min \{k, l\}$, we have $\hat{\mathcal{B}}_{\varepsilon^{k} N} \cap$ $\hat{\mathcal{B}}_{w} \simeq \mathbf{C}^{d} \times V$. Furthermore, $\hat{\mathcal{B}}_{\varepsilon^{k} N} \cap \hat{\mathcal{B}}_{w}=\hat{\mathcal{B}}_{w}$ for $k$ sufficiently large.

Proof. For $1 \leq j \leq l$, let $t_{j} \in \mathbf{C}$ and let $y=x_{\alpha_{1}}\left(t_{1} \varepsilon^{n_{1}}\right) x_{\alpha_{2}}\left(t_{2} \varepsilon^{n_{2}}\right) \ldots x_{\alpha_{l}}\left(t_{l} \varepsilon^{n_{l}}\right)$ with the above notation so that $y \dot{w} \dot{\mathfrak{w}} \hat{\mathfrak{b}}_{0}$ is a point of $\hat{\mathcal{B}}_{w}$. We want to find the conditions on the $t_{j}$ which specify when $y \dot{w} \hat{\mathfrak{b}}_{0} \in \hat{\mathcal{B}}_{\varepsilon^{k} N}$.

It can be seen that any element $M \in \hat{\mathfrak{b}}_{0}$ can be written uniquely in the form $M=M^{+}+M^{-}$where $M^{+} \in \hat{\mathfrak{b}}_{0}, \dot{w}^{-1} M^{+} \dot{w} \in \hat{\mathfrak{b}}_{0}$, and $M^{-}$is a C-linear combination of $\varepsilon^{n} e_{\alpha}$ where $(\alpha, n) \in \tilde{\Delta}(w)$. Thus $\dot{w}^{-1} M \dot{w} \in \hat{\mathfrak{b}}_{0}$ if and only if $M^{-}=0$. Since $y \in \hat{B}_{0}$ and $N \in \hat{\mathfrak{b}}_{0}$, we have $\varepsilon^{k} y^{-1} N y \in \hat{\mathfrak{b}}_{0}$. Hence we can write $\varepsilon^{k} y^{-1} N y$ in the above form as

$$
M^{+}+\sum_{(\alpha, n) \in \tilde{\Delta}(w)} p_{\alpha, n} \varepsilon^{n} e_{\alpha}
$$

Observe that $p_{\alpha, n}$ is a complex polynomial in the variables $t_{j}$ for which $n_{j} \leq n-k$ and $p_{\alpha, n}=0$ if $n<k$. This follows from the fact that if $t \in F$ and $\beta \in \Delta$, then $x_{\beta}(t)$ acting by conjugation on $\hat{\mathfrak{g}}$ acts as a matrix (with respect to a basis coming from $\mathfrak{g}$ ) with entries that are complex polynomials in $t$.

Now $y \dot{w} \hat{\mathfrak{b}}_{0} \in \hat{\mathcal{B}}_{\varepsilon^{k} N}$ if and only if $\varepsilon^{k} \dot{w}^{-1} y^{-1} N y \dot{w} \in \hat{\mathfrak{b}}_{0}$. And this is true if and only if $p_{\alpha, n}=0$ for all $(\alpha, n) \in \tilde{\widetilde{\Delta}}(w)$. Hence

$$
\hat{\mathcal{B}}_{\varepsilon^{k} N} \cap \hat{\mathcal{B}}_{w}=\left\{\left(t_{1}, \ldots, t_{l}\right) \mid p_{\alpha, n}=0 \text { for all }(\alpha, n) \in \tilde{\Delta}(w)\right\} .
$$

But $p_{\alpha, n}$ is not a function of all $t_{j}$. In fact, let $t_{j_{1}}, \ldots, t_{j_{h}}$ be a list of those $t_{j}$ which appear in at least one $p_{\alpha, n}$. Let

$$
V=\left\{\left(t_{j_{1}}, \ldots, t_{j_{h}}\right) \mid p_{\alpha, n}=0 \text { for all }(\alpha, n) \in \tilde{\Delta}(w)\right\} .
$$

Thus if $V \neq \emptyset$, then $\hat{\mathcal{B}}_{\varepsilon^{k} N} \cap \hat{\mathcal{B}}_{w} \simeq \mathbf{C}^{l-h} \times V$.

Let $(\beta, m) \in \tilde{\Delta}(w)$ be such that $m=\max \{n \mid(\alpha, n) \in \tilde{\Delta}(w)\}$. Then $t_{j}$ does not contribute to any $p_{\alpha, n}$ if $n_{j}>m-k$. We now consider the two cases $0<k \leq m$ 
and $k>m$ separately. If $0<k \leq m$, then $(\beta, m), \ldots,(\beta, m-k+1)$ belong to $\tilde{\Delta}(w)$ but do not contribute to any $p_{\alpha, n}$. Hence in this case, the number of $t_{j}$ such that $n_{j}>m-k$ is at least $k$, i.e., $l-h \geq k$. On the other hand, if $k>m$, then all $p_{\alpha, n}$ are zero, i.e., $\hat{\mathcal{B}}_{\varepsilon^{k} N}=\hat{\mathcal{B}}_{w}$. In either case, $\hat{\mathcal{B}}_{\varepsilon^{k} N} \cap \hat{\mathcal{B}}_{w}$ has the desired form and $\hat{\mathcal{B}}_{\varepsilon^{k} N} \cap \hat{\mathcal{B}}_{w}=\hat{\mathcal{B}}_{w}$ whenever $k>m$. This completes the proof.

\section{MAin RESUlT}

We return to the case where $N \in \hat{\mathfrak{g}}$ is nilpotent. We can now compute the singular homology of $\hat{\mathcal{B}}_{N}$ with integer coefficients.

Theorem. For any nilpotent element $N \in \hat{\mathfrak{g}}$, we have $H_{*}\left(\hat{\mathcal{B}}_{N}\right) \simeq H_{*}(\hat{\mathcal{B}})$.

Proof. Without loss of generality, we can assume $N \in \hat{\mathfrak{b}}_{0}$. Fix a nonnegative integer $i$. We wish to show that $H_{i}\left(\hat{\mathcal{B}}_{N}\right) \simeq H_{i}(\hat{\mathcal{B}})$. Since $\hat{\mathcal{B}}_{N}$ and $\hat{\mathcal{B}}_{\varepsilon^{2 k} N}$ are homeomorphic by Lemma 1, it will be enough to show that $H_{i}\left(\hat{\mathcal{B}}_{\varepsilon^{2 k} N}\right) \simeq H_{i}(\hat{\mathcal{B}})$ for some positive integer $k$.

By Lemma 2, we can choose $k$ so that for all $w \in \hat{W}$ with $l(w) \leq i$, we have $\hat{\mathcal{B}}_{\varepsilon^{2 k} N} \cap \hat{\mathcal{B}}_{w}=\hat{\mathcal{B}}_{w}$. Furthermore, by increasing $k$ if necessary, we can also guarantee that if $l(w)>i$, then either $\hat{\mathcal{B}}_{\varepsilon^{2 k} N} \cap \hat{\mathcal{B}}_{w}=\emptyset$ or for some complex variety $V$, we have $\hat{\mathcal{B}}_{\varepsilon^{2 k} N} \cap \hat{\mathcal{B}}_{w} \simeq \mathbf{C}^{i+1} \times V$. Let us introduce some more notation. For $w \in \hat{W}$, let

$$
Y_{w}=\hat{\mathcal{B}}_{\varepsilon^{2 k} N} \cap \hat{\mathcal{B}}_{w}
$$

and

$$
X_{n}=\bigcup_{\substack{w \in \hat{W} \\ l(w) \leq n}} Y_{w} .
$$

Note that $X_{n}$ is closed in $\hat{\mathcal{B}}_{\varepsilon^{2 k} N}$ and $\hat{\mathcal{B}}_{\varepsilon^{2 k} N}=\underline{\lim } X_{n}$ as topological spaces. Clearly

$$
X_{n}=X_{n-1} \cup Y_{w_{1}} \cup Y_{w_{2}} \cup \cdots \cup Y_{w_{h}}
$$

where $\left\{w_{1}, w_{2}, \ldots, w_{h}\right\}$ are the elements of $\hat{W}$ of length $n$. Let $X_{n, 0}=X_{n-1}$ and for $1 \leq j \leq h$, define $X_{n, j}$ inductively to be $X_{n, j-1} \cup Y_{w_{j}}$. Thus $X_{n, h}=X_{n}$. Note that $X_{n, j-1}$ is closed in $X_{n, j}$.

Because $k$ is chosen so that $Y_{w}=\hat{\mathcal{B}}_{w}$ for all $w \in \hat{W}$ with $l(w) \leq i$, we see that for $0 \leq r \leq 2 i$

$$
H_{r}\left(X_{i}\right) \simeq H_{r}(\hat{\mathcal{B}}) .
$$

In particular, this holds for $r=i$.

Now suppose we can establish that for all $n \geq i+1$ the inclusion of $X_{n-1}$ in $X_{n}$ induces an isomorphism of $H_{i}\left(X_{n-1}\right)$ with $H_{i}\left(X_{n}\right)$. Then since $\hat{\mathcal{B}}_{\varepsilon^{2 k} N}=\underline{\lim } X_{n}$, it will follow that

$$
H_{i}\left(\hat{\mathcal{B}}_{\varepsilon^{2 k} N}\right) \simeq H_{i}\left(X_{i}\right) \simeq H_{i}(\hat{\mathcal{B}})
$$

which would complete the argument.

So assume $n \geq i+1$. Let $H_{*}^{B M}(X)$ denote the Borel-Moore homology with integer coefficients of the space $X[1]$. Then the following exact sequence holds:

$$
\cdots \longrightarrow H_{i+1}^{B M}\left(Y_{w_{j}}\right) \longrightarrow H_{i}^{B M}\left(X_{n, j-1}\right) \longrightarrow H_{i}^{B M}\left(X_{n, j}\right) \longrightarrow H_{i}^{B M}\left(Y_{w_{j}}\right) \longrightarrow \cdots
$$


since $X_{n, j-1}$ is closed in $X_{n, j}$. By the assumption on $n$ and the choice of $k$, we have either $Y_{w_{j}}=\emptyset$ or $Y_{w_{j}} \simeq \mathbf{C}^{i+1} \times V$. In the latter case, since $H_{r}^{B M}\left(\mathbf{C}^{i+1}\right)$ vanishes for $r<2(i+1)$, the Künneth theorem implies that both $H_{i+1}^{B M}\left(Y_{w_{j}}\right)$ and $H_{i}^{B M}\left(Y_{w_{j}}\right)$ vanish. Hence in either case, the inclusion of $X_{n, j-1}$ in $X_{n, j}$ induces an isomorphism of $H_{i}^{B M}\left(X_{n, j-1}\right)$ with $H_{i}^{B M}\left(X_{n, j}\right)$. Since this holds for all $j$, we have the desired isomorphism of $H_{i}^{B M}\left(X_{n-1}\right)$ with $H_{i}^{B M}\left(X_{n}\right)$ for all $n \geq i+1$. Finally, we note that the isomorphism is valid in singular homology because the $X_{n}$ are compact and triangulable (being projective), so there is no distinction between Borel-Moore and singular homology.

\section{Generalization}

A similar result holds for the partial affine flag manifolds. Let $\hat{\mathfrak{p}}_{0}$ be a parahoric subalgebra of $\hat{\mathfrak{g}}$ and let $\hat{\mathcal{P}}$ be the space of subalgebras conjugate to $\hat{\mathfrak{p}}_{0}$. For a nilpotent element $N \in \hat{\mathfrak{g}}$, we let $\hat{\mathcal{P}}_{N}$ be the subspace of $\hat{\mathcal{P}}$ consisiting of parahoric subalgebras conjugate to $\hat{\mathfrak{p}}_{0}$ which contain $N$. Then $H_{*}\left(\hat{\mathcal{P}}_{N}\right) \simeq H_{*}(\hat{\mathcal{P}})$. The proof is similar to the above case.

\section{ACKNOWLEDGEMENT}

I would like to thank G. Lusztig for suggesting this problem and giving many helpful comments.

\section{REFERENCES}

1. A. Borel and J. C. Moore, Homology theory for locally compact spaces, Michigan Math. J. 7 (1960), 137-159. MR 24:A1123

2. N. Iwahori and H. Matsumoto, On some Bruhat decomposition and the structure of the Hecke rings of p-adic Chevalley groups, Inst. Hautes Etudes Sci. Publ. Math. 25 (1965), 5-48. MR 32:2486

3. N. Jacobson, Lie Algebras, Dover Publications, Inc., New York, 1979, p. 100. MR 80k:17001

4. V. Kac, Constructing groups associated to infinite-dimensional algebras, in Infinite Dimensional Groups with Applications, MSRI Publications, Springer-Verlag, Berlin, 1985, pp. 198199.

Department of Mathematics, Massachusetts Institute of Technology, Cambridge, MASSACHUSETtS 02139

E-mail address: esommers@math.mit.edu 